\title{
Interconnection of antitoxic and antioxidant systems of the organism under the action of natural low molecular complex - fungidol
}

\author{
Natalia Igorevna Kurguzova, Anatoliy Ivanovich Bozhkov", Yuriy Viktorovich Nikitchenko, \\ Mohammad Ali Yousef Al Begai, Anatoliy Vladimirovich Goltvyansky, \\ Mohammad Morshed Ayed Alsardia, Andrew Anatolievich Bozhkov
}

Biology Research Institute of V. N. Karazin Kharkov National University, Kharkov, Ukraine

Email address:

bozhkov@univer.kharkov.ua (A. I. Bozhkov)

\section{To cite this article:}

Natalia Igorevna Kurguzova, Anatoliy Ivanovich Bozhkov, Yuriy Viktorovich Nikitchenko, Mohammad Ali Yousef Al Begai, Anatoliy Vladimirovich Goltvyansky, Mohammad Morshed Ayed Alsardia, Andrew Anatolievich Bozhkov. Interconnection of Antitoxic and Antioxidant Systems of the Organism under the Action of Natural Low Molecular Complex - Fungidol. American Journal of Biomedical and Life Sciences. Special Issue: Mechanisms of Protection against Oxidative Stress. Vol. 2, No. 6-1, 2015, pp. 25-32.

doi: $10.11648 /$ j.ajbls.s.2014020601.15

\begin{abstract}
The influence of the natural complex of low molecular weight compounds (fungidol) on antitoxic and antioxidant systems in animals was investigated. It was demonstrated that the introduction to the experimental animals of fungidol in doses a $0.05 \mathrm{ml}$ per $100 \mathrm{~g}$ of body weight increases antioxidant enzymes activity in serum and liver, decreases the content of lipids hydroperoxides and other products of free radical reactions. The components of fungidol antioxidant activity correlated with the stability of membranes of erythrocytes in these animals. In the experimental model the toxic effect of copper sulfate was investigated. The increase in the activity of prooxidant system induced by fungidol correlated with its antitoxic effect.
\end{abstract}

Keywords: Antioxidant System, Antitoxic Activity, Prooxidant Activity, Fungidol

\section{Introduction}

Russian biologist A.I. Bach formulated the peroxide theory of oxidation in 1897, in which the presence of peroxides in living cells was predicted, which was an important step in understanding mechanisms of oxygen activation. Bach subsequently extended the peroxide theory to processes of biological oxidation in 1904. Late on, it has been experimentally confirmed that in biological systems free radicals are indeed generated in the course of a metabolism. These highly reactive molecules, as a rule, have a very short period of life and can oxidize a wide variety of macromolecules in living organism. The intensity of free radical generation in biological systems was estimated by products of free radical reactions being formed i.e. quantity of the oxidized lipids, carbonylated proteins, aldehydes, etc [1-3].

The free-radical theory of ageing first proposed by Harman [4] and Emanuel [5] in 1956-1958 became a strong incentive for research of free radical products in biological systems.
This theory has found a large number of supporters and experimental proof [6-8].

Since then, a large body of experimental data has been accumulated and has led to a formation of a special scientific discipline - free radical biology. It was found that the induction of free radical production, that can further lead an oxidative stress, is nonspecific reaction to a wide range a stress factors [9-10].

The majority of pathologies are to some extent connected with development of an oxidative stress, and at a certain stage of studies it seemed that the problem pathogenesis, specifically age-related pathologies can be simply solved, in particular, using endogenous and exogenous antioxidants. Antioxidants which are numerous and it is possible to "regulate" quantitative manifestations of an oxidative stress and, as a result, to eliminate pathologies [11].

However, despite certain successes in anti-radical therapy, the expectations have not come true fully, and received results on application of antioxidants are rather inconsistent [12-13].

To explain this, V.K. Koltover suggested that the 
antioxidant system of the living organism is so effective that it cannot be significantly altered by rather small amount of exogenous antioxidants [14].

Moreover, significant decrease in free radical production in an organism as can lead to negative metabolic effects, therefore the products of free radical reactions carry out physiologically important function of signalling [15-17].

It is possible to conclude that the prooxidant-antioxidant system is one of regulatory systems of an organism which changes not only in response to powerful stress factors, physical activities, etc., but also to physiological stimuli, such as special features of a food and even strong physical activities [18-20]. However, there is not clear the contribution of the prooxidant system to development of pathologies and responses to a stress stimulus, i.e. is response of prooxidant system the cause or consequence of pathological processes. Hence, the essence of a problem of the contribution of pro-oxidant system in development of pathologies, processes of ageing and adaptation mechanisms most likely is determined by interaction with other functional systems of an organism. In this interaction a ratio between various functional systems of an organism are more important than the quantitative production level of free radicals. Therefore, to prevent the pathological processes it is necessary not only to control regulation of the production of free radicals and their interception by antioxidants, but also to control a connections of prooxidant-antioxidant system with other systems which provide anti-toxic, anticancerogenic, anti-mutagenenic, anti-inflammatory and other functions of an organism which are responsible for regulatory and homeostatic functions. Based on this, we hypothesize that any study of the efficiency modulation of prooxidantantioxidant system should be carried out in connection with assessment of other systems, in particular the anti-toxic system. For modulation of anti-toxic and antioxidant function, we have developed a natural complex of low-molecular weight organic components - fungidol, which can regulate antioxidant and anti-toxic functions of an organism. The aim of the present study was to investigate the inter-relationship between antitoxic and antioxidant systems in animal model of intoxication of an organism by copper-ions by using natural complex fungidol as modulator.

\section{Materials and Methods}

The experiments were done on three-months-old Wistar rat males. The experiments were done in concordance with the bioethics rules (European Convention for the Protection of the Vertebrata used for experimental and scientific aims, Strasburg, 1986).

All the animals (40) were divided in four groups, 10 animals in each group. The first group: the intact control animals was injected normal saline solution in dose $0.5 \mathrm{ml}$ per $100 \mathrm{~g}$ of body weight;

- the second group - animals to which was injected the copper sulfate intraperitoneally in dose $1 \mathrm{mg}$ per $100 \mathrm{~g}$ body weight three times (once per 48 hours) in the morning before feeding. It corresponds to $\mathrm{LD}_{30}$. The interval between copper sulfate additions was 48 hours.

- the third group - animals to which was injected the copper sulfate intraperitoneally in the same scheme, but 24 hours before the injection of copper sulfate the fungidol was added per os in dose $0.5 \mathrm{ml}$ per $100 \mathrm{~g}$ of body weight.

- the fourth group - animals to which was added the fungidol per os in dose $0.5 \mathrm{ml}$ per $100 \mathrm{~g}$ of body weight. The interval between copper sulfate additions was 48 hours.

Twenty four hours after the last copper sulfate addition the animals were taken in the experiment.

The body weight, rectal temperature, and forced swimming test (time of swimming with loading) [21] was applied to all animals. The rectal temperature was measured by TW 2-193 2 Microtherma T Hand Held Thermometer (BRAINTREE SCIENCIFIC, INC., USA).

The determination of antioxidant activity in model system in vitro was done as described [22]. The determination of antiradical activity in model system in vitro was done as described [23].

Preparation of mitochondria, and microsomes. Mitochondria and microsomes was isolated by differential centrifugation. The isolated mitochondria were suspended in the medium: $0.3 \mathrm{M}$ sucrose $-10 \mathrm{mM}$ Tris $-\mathrm{HCl}$ buffer, $\mathrm{pH} 7.4$ and their concentration was adjusted to $60-80 \mathrm{mg} / \mathrm{ml}$ total protein. The isolated microsomes were suspended in $125 \mathrm{mM}$ $\mathrm{KCl}, 10 \mathrm{mM}$ Tris -HCl-buffer, $\mathrm{pH}$ 7.4.

Glutathione peroxidase (GP, EC 1.11.1.9) activity was determined in cytosolic fractions, and liver mitochondria serum by spectrophotometer at $340 \mathrm{~nm}$ by method [24] in 50 $\mathrm{mM} \mathrm{K}^{+}, \mathrm{Na}^{+}$- phosphate buffer $(\mathrm{pH}$ 7.4) containing $1 \mathrm{mM}$ EDTA, $0.15 \mathrm{mM}$ NADPH, 1 unit of yeast glutathione reductase, $0.2 \%$ Triton $\mathrm{X}-100$ and $3 \mathrm{mM} \mathrm{Na}$ azide to inhibit KAT. $1.2 \mathrm{mM}$ cumene hydroperoxide and $0.4 \mathrm{mM}$ hydrogen peroxide were added. Incubation temperature was $37^{\circ} \mathrm{C}$. The activity was expressed in nmol NADPH/min per $1 \mathrm{mg}$ of protein or $1 \mathrm{ml}$ of serum considering a molar extinction coefficient $6.22 \cdot 10^{3} \cdot \mathrm{M}^{-1} \mathrm{~cm}^{-1}$.

The glutathione-S-transferase (GT EC 2.5.1.18) activity was measured in liver cytosol and mitochondria by spectrophotometer at $340 \mathrm{~nm}$ [25] in a medium containing $0.1 \mathrm{M} \mathrm{K}^{+}$phosphate buffer, $\mathrm{pH} 6.5,1 \mathrm{mM}$ 1-chloro-2,4dinitrobenzene, $5 \mathrm{mM}$ glutathione $(\mathrm{GSH}), 0.2 \%$ Triton $\mathrm{X}$ 100. The incubation temperature was $37^{\circ} \mathrm{C}$. The activity was calculated using a molar extinction coefficient $9.6 \cdot 10^{3} \mathrm{M}^{-}$ ${ }^{1} \mathrm{~cm}^{-1}$.

Determination of lipid hydroperoxides. Hydroperoxides content in subcellular fractions was determined by the method of Ohkawa et al. [26] and in the serum by the method of Asakawa et al. [27]. The content of lipid hydroperoxides was expressed in equivalent quantities of malone dialdehyde (MDA) per $1 \mathrm{mg}$ of plasma or serum.

All the experiments were repeated no less than three times and the data obtained were analyzed statistically by Wilcoxon-Mann-Witney test. 


\section{Results}

Estimation of toxic effect of copper-ions and anti-toxic action of fungidol.

Copper sulfate was injected to rats intraperioneally at the intervals of 48 hours between injections at a dose of $1 \mathrm{mg}$ per $100 \mathrm{~g}$ animal body weight which corresponds to lethal doses of $30-33 \mathrm{mg} / \mathrm{kg}$ (LD 30-33) for rats of the Wistar strain [28].

As presented in Table 1, it was found that five days after first injection the experimental animals lost body mass while in control group it increased by $5 \mathrm{~g}$.

Moreover, the body temperature during the experiment in the animals of control group maintained at level of standard control $\left(37.03 \pm 0.13^{\circ} \mathrm{C}\right)$, while the body temperature of the copper sulfate treated animals was lower almost on $1^{\circ} \mathrm{C}$ that indicates a decrease in the general metabolism in these animals (Table 1).

Table 1. Changes in body weight and body temperature, and physical capacity indicator (time of swimming with loading) after 5 days from the start of the experiment with addition of copper sulfate in different experimental groups. Each group consisted of 10 animals.

\begin{tabular}{llll}
\hline \multirow{2}{*}{ Group of animals } & Parameter & \\
\cline { 2 - 4 } & Body mass change from initial, \% & Body temperature, ${ }^{\circ} \mathbf{C}$ & Time of swimming, sec \\
\hline Control & $105.0 \pm 1.5$ & $37.03 \pm 0.3$ & $900.0 \pm 25.5$ \\
After intoxication & $98.0 \pm 1.2^{*}$ & $36.13 \pm 0.3^{*}$ & $167.0 \pm 10.1^{*}$ \\
$\begin{array}{l}\text { Intoxication against the background of } \\
\text { fungidol addition }\end{array}$ & $115.0 \pm 2.0^{*}$ & $37.6 \pm 0.3$ & $267.0 \pm 15.2^{*}$ \\
\hline
\end{tabular}

Asterisk (*) - the significant difference compared with the control $(\mathrm{P}<0.05)$.

A reliable physiological indicator of toxicity of copperions in animals is the alteration of ability to generate the metabolic energy required to perform physical activity.

In this study, the alteration of ability to perform physical activity by rats was judged according to the standardized test - swimming time with cargo [21].

It appeared that the animals that were administered with copper sulfate, in 5 days after the last injection lost their ability to swim and to stay at the water surface in 5 times faster in comparison with control animals (Table 1).

Consequently, a copper toxicity in rats was manifested by growth oppression and weight loss, decrease in body temperature, and the alteration of the ability to perform physical activities that especially demonstrated in the swimtest in the pool with cold water $\left(14-15^{\circ} \mathrm{C}\right)$.

For estimating the anti-toxic property of fungidol, the animals received fungidol per os 24 hours prior to injection of copper sulfate.

It was found that in that case the animal body mass increased and even exceeded control group (Table 1).

Although a body temperature of the rats administered with copper sulfate together with fungidol did not differ significantly from control animals, but "the time of swimming" of these animals increased in comparison with rats that received only copper sulfate by $60 \%$, and it was less in 3 times compared with control animals (Table 1).

Therefore, fungidol at a dose of $0.5 \mathrm{~mL}$ per $\mathrm{kg}$ of body weight has a pronounced anti-toxic effect.

The large doses of copper sulfate (2.5-3.0 mg per $100 \mathrm{~g}$ of body weight) can cause an acute toxicity and death of experimental animals during 1-6 hours after injection [29].

However, the pre-injections of several small doses of the copper sulfate, that caused chronic toxicity, can provide resistance to the subsequent injection of a lethal dose, so the hormesis effect was manifested [30].

For inducing the hormesis effect, the experimental rats were pre-injected with 3 small doses of the copper sulfate as described in Materials and Methods section.
The five days later after the last pre-injection of small dose of copper sulfate to the three groups of experimental animals the lethal dose of copper sulfate was injected, and the number of animals that survived three days after the taking lethal dose was determined.

It was found that all animals of control group died within 1-6 hours after lethal injection of $2.5 \mathrm{mg}$ per $100 \mathrm{~g}$ of body weight of copper sulfate (Fig. 1).

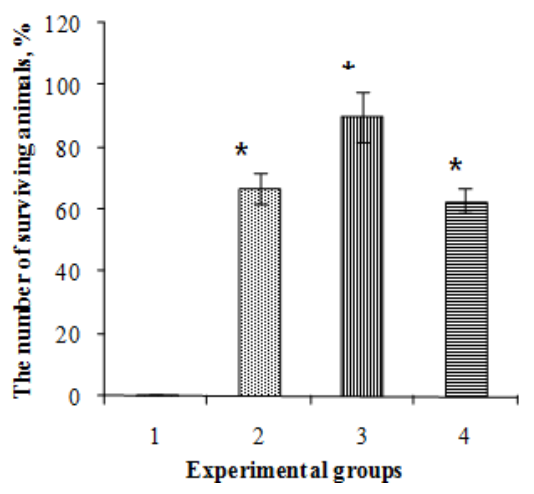

Figure 1. The number of survived animals in three days after the administration of copper sulfate, $2.5 \mathrm{mg} / 100 \mathrm{~g}$ body weight (in percent). A control group of animals which received normal saline solution (1), a group of animals, which had previously been injected three times a copper sulfate 1 mg / $100 \mathrm{~g}$ body weight (2), a group of animals which were administered copper sulfate with preliminary administration of fungidol (3) and group of animals which had been administered fungidol (4).

Strikingly, in the group of rats pre-injected with small doses of copper sulfate $67 \%$ of animals survived, whereas, in the group of rats pre-injected with copper sulfate together with fungidol $90 \%$ of animals survived, i.e. by $23 \%$ more compared to the animals that received only copper sulfate.

These results indicate that the hormesis effect was manifested (Fig. 1).

In case when animals received only fungidol three times at the intervals between injection of 48 hours and after that a large dose of copper sulfate $(2.5 \mathrm{mg}$ per $100 \mathrm{~g}$ of body weight) survived more than $60 \%$ of animals (Fig. 1). 
Therefore we conclude that fungidol have antidotal property, and it can reduce toxic effect of copper sulfate in an organism and increase copper-induced effect of hormesis.

The characteristics of pro- and antioxidant system of experimental animals.

Adding fungidol $(2.5 \mu \mathrm{L}$ to $1 \mathrm{~mL}$ of the reaction solution) to the model system slowed down the oxidation of yolk lipoproteins by $28 \%$, and when the amount of fungidol in the reaction increased $(5-10 \mu \mathrm{L}$ to $1 \mathrm{~mL})$ the antioxidant activity increased almost linearly in dependence of its addition (Fig. 2A). For comparison, the antioxidant activity of fungidol appears to be similar to that of a well known flavonoid quercetin, however, the fungidol activity significantly exceeded of an antioxidant activity of routine.

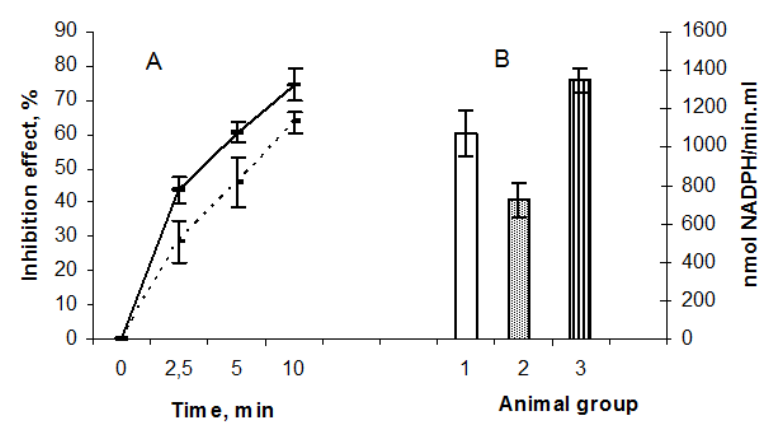

Figure 2. A: antioxidant (---) and antifree-radical (-) activity of fungidol in vitro in percent to control.

B: glutathione peroxidase activity in control group of animals (1); animals, which received the copper sulfate (2); and animals, treated by fungidol which received the copper sulfate (3).

* - $\mathrm{P}<0,05$ in comparison to control; ** $-\mathrm{P}<0,05$ in comparison to control and group of animals, which received the copper sulfate.

It was found that the components of fungidol can efficiently intercept of $\mathrm{OH}$ - radicals (Fig. 2A).

So, adding fungidol $(2.5 \mu \mathrm{L}$ to $1 \mathrm{~mL}$ of the reaction solution) to the model system inhibited production of $\mathrm{OH}$ radicals by $43 \%$, and when increased amount added $(10 \mu \mathrm{L}$ to $1 \mathrm{~mL}$ ) by $74.6 \%$. For comparison, a well known quencher for $\mathrm{OH}$ radicals - ethanol at a concentration of $0.5 \mathrm{mg} / \mathrm{mL}$ inhibits generation of $\mathrm{OH}$ radicals by $67.5 \%$.

Therefore, fungidol contains the pronounced antioxidant and anti-radical properties in vitro and this may, in part, explain its protective effect against toxicity of copper sulfate.

The estimation of activity of the key antioxidant enzyme of glutathione system - glutathione peroxidase (GP) in blood serum demonstrated that three sequential pre-injection of a small doses of the copper sulfate reduced the GP enzyme activity by $33 \%$ when compared with control (Fig. 2 B).

Whereas, in case when copper sulfate was injected together with administration of fungidol, the glutathione reductase (GR) activity was $86 \%$ higher when compared with the enzyme activity from blood serum of animals that received only copper sulfate, and it was by $25 \%$ above control animals (Fig. 2 B).

These results demonstrate convincingly that fungidol contains the pronounced antioxidant activity both in vitro and in vivo.

It is well known that the liver is the main place of accumulation of exogenous copper and other toxic substances in rats. Therefore, next we determined the effect of fungidol on some indicators of prooxidat and antioxidant systems in mitochondria and microsomes, which are the major sources of free radical in a liver.

Effect of fungidol on prooxidant-antioxidant balance in mitochondria and microsomes from liver.

The GP activity in rat liver mitochondria after administration of fungidol increased in 4.9 times as compared with control, and in fraction of microsomes - in 1.7 times (Fig. 3).

The glutathione-transferase (GT) activity also increased after administration of fungidol, however, to a much lesser extent, i.e., in mitochondria by $43 \%$, and in fraction of microsomes by $33 \%$ (Fig. 3).

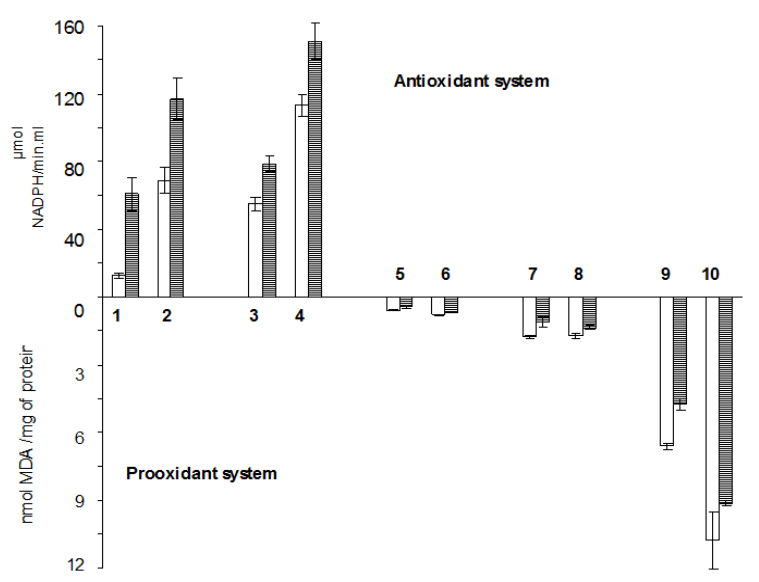

Figure 3. The activity of glutathione peroxidase in liver mitochondria in control (口), animals treated with fungidol (ロ) (1), glutathione transferase activity in mitochondria in the same groups of animals (2), glutathione peroxidase in rat liver microsomes (3) and content of glutathione in microsomes (4).

Contents of lipid hydroperoxides in mitochondria in control ( $\square$ ), animals treated with fungidol (घ) in mitochondria (5), spontaneous peroxidation products in the mitochondria of the same groups of animals (6), lipid peroxidation in mitochondria acrobat-induced lipid peroxidation of the same animals (7), and the same parameters in the fraction of microsomes, respectively $(8,9,10)$.

Thus, these results demonstrate that fungidol stimulate enzymatic activity of GP and GT in rat liver mitochondria and microsomes.

The spontaneous and an ascorbate-induced lipid peroxidation in the animals that received fungidol was decreased as compared with control. So, the lipid hydroperoxide content in mitochondria and microsomes decreased by $21 \%$ and $12 \%$ as compared with control. In same time, spontaneous lipid peroxidation (SLPO) decreased by $30 \%$ and $22 \%$, respectively, and an ascorbate-induced lipid peroxidation (ALPO) also was significantly decreased as compared with control (Fig. 3).

In summary we conclude that fungidol by activating the cellular antioxidant system can provide reduction of products 
of free radical reactions, and stimulate the resistance of the organism to the toxic effect of copper.

The protective effect of fungidol on the erythrocyte membranes.

As it is known, free radicals in biological systems induce typical chain process with the pronounced branching. Its development may be interrupted for two main reasons: to intercept components of the antioxidant system with adaptive further restructuring of metabolism or cell death and its subsequent elimination. When cell death is not compensated by proliferative processes, it can be formed new metabolic relationships between tissues that may be manifested in different pathologies depending on the localization of breach in the hierarchical structure of a biological system, and the retention time of these changes.

To investigate possible mechanisms of copper-induced animal death we proceeded from the fact that the death of animals in acute intoxication with copper occurs as a result of inhibition of the function of blood circulation, spasm of blood vessels of the liver and kidneys. One of the possible molecular mechanisms of acute toxic effect of high concentrations of copper ion is the induction of oxidative processes of the cell membranes, primarily erythrocyte membranes. If so, the oxidation of erythrocyte membrane components can lead to cell death, reduction in amount of the bloodstream and the remaining cells can lose their functional activity, and this leads to respiratory failure and death of the animals.

Determination of the number of erythrocytes in the control group of animals showed that it was $(6.1 \pm 0.42)$ million $/ \mu 1$. In the case if the animals received thrice copper sulfate in small doses - LD30, the number of erythrocytes was $32 \%$ less than the reference level (Fig. 4).

In that case, if the same dose of copper sulphate was administered on the background of fungidol, the number of erythrocytes did not differ from the control level (Fig. 4). Erythrocytes didn't differ by the morphological characteristics from red blood cells of the control animals. These results suggest that the components of fungidol are able to provide "protection" of erythrocyte membranes from the toxic effect of copper ions.

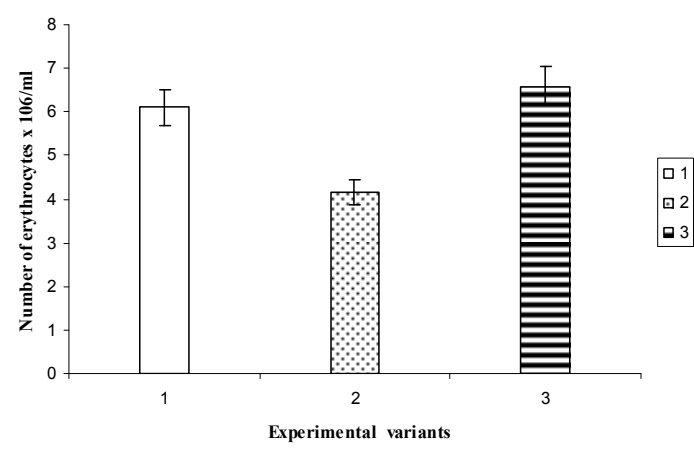

Figure 4. The number of erythrocytes in the group of control animals (1), the animals, to which was injected the copper sulfate (2) and animals, to which was injected the copper sulfate after fungidol treatment (3).

* $-\mathrm{P}<0.05$ compared with control.

\section{Discussion}

According to the degree of toxicity the substances are divided into 4 groups: overly-toxic - $\mathrm{LD}_{50}=15 \mathrm{mg} / \mathrm{kg}$; high-toxic $\mathrm{LD}_{50}=150 \mathrm{mg} / \mathrm{kg}$; moderately toxic, $\mathrm{LD}_{50}=$ $1500 \mathrm{mg} / \mathrm{kg}$; low-toxic - LD $501500 \mathrm{mg} / \mathrm{kg}$. According to this classification the copper sulfate is highly toxic compound.

The toxicity may depend on: 1. - the structure and concentration of the substance; 2 - metabolic characteristics of biological object (initial resistance). On this basis in the development of antidotes can be used at least two approaches, which can be called chemical and biological, and their combinations thereof may not be excluded.

A chemical approach is based on the use of various compounds capable to form complexes with toxicants, particularly with heavy metal ions. Thus, salts of ethylenediaminetetraacetic acid (EDTA), which is a good chelating agent [29], or compounds that are donors of SH groups are the most common antidotes for heavy metal ions [30].

The biological approach may be based on the use of such biologically active compounds which act on regulatory systems of metabolism, modifying it by such way, that it will be able to provide the hormesis but not toxic effect.

Previously, it was shown that the hormesis effect of copper ions is determined by cooperative change in many, if not all protective systems of the body, which are characterized by the emergence [30].

It can be assumed that the cooperative changes or modulation of metabolic elements providing a manifestation of antitoxic, anticarcinogenic, and even antimutagenic properties are associated with the antioxidant, immune and neuroendocrine systems of the organism.

We believe that the antitoxic effect of high concentrations of copper will be manifested to the greatest extent in the cooperatively change several regulatory systems that protectorganism: 1 - binding and inactivation of toxic products and their metabolites; 2 - providing their biotransformation and 3 - deposition or removal of the detoxification products out of the body.

To achieve this, we have developed a complex of lowmolecular components of natural origin.

Its main components are represented by oligosaccharides (66\%), low molecular weight proteins (M.W. less than 20 $\mathrm{kDa}, 23 \%)$ and lipids (10\%). In addition, it contains free amino acids (valine is 13 , cysteine -16 , methionine -11 , leucine - 10, arginine - 4, isoleucine - 4, threonine -3 and lysine $-1 \%$ of all amino acids) and vitamins $\left(\mathrm{B}_{1}-0.38 \mathrm{mg} / \mathrm{l}\right.$, $\mathrm{B}_{2}-3.24 \mathrm{mg} / \mathrm{l}$ and $\left.\mathrm{PP}-8.3 \mathrm{mg} / \mathrm{l}\right)$.

Such a wide range of biologically active compounds can provide soft complex action on a number of regulatory systems.

Thus, the administration of of copper sulfate in lethal dose to the animals that preliminary received fungidol provides survival to $60 \%$ of the animals, whereas there was $100 \%$ lethality in control group. Furthermore, administration of low 
doses of copper sulfate $\left(\mathrm{LD}_{30}\right)$ on a background of fungidol provides not only survival after subsequent lethal dose and but the increase of efficiency, body temperature and growth rate of these animals.

The mechanisms of antitoxic action of fungidol are of undoubted interest.

It is known that the acute toxicity of copper sulfate is associated with inhibition of respiratory function, functioning circulatory and excretory systems [31]. The degree of violation of these vital functions is correlated with the functional activity of the red blood cells. It was found that after three consecutive administrations of copper sulphate in $\mathrm{LD}_{30}$ doses erythrocyte number decreased by $30 \%$, and their morphology also changed indicating the change of their functional activity. We can assume that after the administration of copper sulfate in large doses $\left(\mathrm{LD}_{50}-\mathrm{LD}_{100}\right)$, these changes will be more pronounced and can be fatal.

It is known that erythrocyte hemolysis in the case of copper ions is induced by lipid peroxidation of membranes

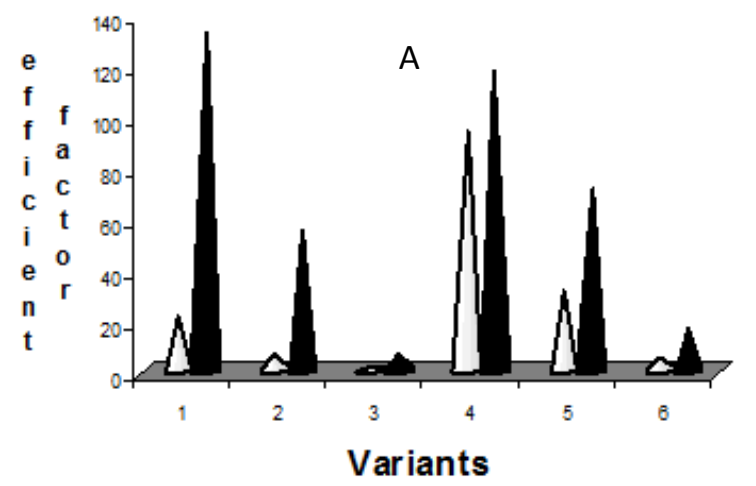

components.

In the case of administration fungidol before copper sulfate, the content of erythrocytes and functional activity even after the administration of copper sulfate didn't differ from control (Fig. 3). This is because the components of fungidol possessing antioxidant and antiradical properties, and inducing an increase in GP activity and GT, lead to the cooperative rearrangements of the whole metabolism which occur in increasing of the resistance of animals to lethal doses of copper sulfate.

Thus, in the animals that received fungidol the GP and GT activity was significantly increased and on this background content of hydroperoxides in mitochondria and microsomes was reduced (Fig. 3). If we assume that the amount of hydroperoxides is determined by the activity of GP, GT and other antioxidant enzymes and to determine the relationship between these parameters, it is possible to suggest a conditional coefficient of the effectiveness of antioxidant protection.

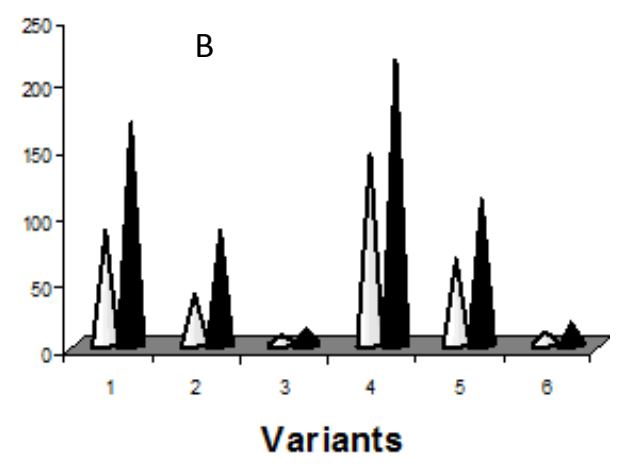

Figure 5. The coefficient of relative efficiency of the antioxidant system, presented as the ratio of the GP /LHP (1), GP / SLPO (2), GP / ALPO (3), GT /LHP (4), GT / ALPO (6) in mitochondria (A) and the same in microsomes (B).

It was found that fungidol effectively increased antioxidant protection system at the level of mitochondria. Thus, the ratio of GP to lipid hydroperoxides in experimental group of animals treated by fungidol was 5-8 times higher compared with the control (Fig. 5). It should be noted that all of the studied indicators of antioxidant protection in liver microsomes and mitochondria from animals that received fungidol were higher from 50 to $780 \%$ compared with the control (Fig. 5).

Such marked differences in the prooxidant activity of the enzymes of glutathione cycle between control and experimental animals may indicate cooperative changes of the whole metabolism that underlies the resilience of erythrocytes and other types of cell membranes and eventually the stability of the whole organism.

As it is known, glutathione peroxidase, glutathione transferase and glutathione reductase are key enzymes glutathione cycle [32]. Glutathione is one of the main antioxidant components of biological systems. Its content in the cell is very high and can reach up to $5 \mathrm{mM}$ : Perhaps this is the only antioxidant that is cyclically transferred from the primary to the oxidized form and its level varies in all cases the displacement relations in pro / antioxidant system (Fig. 6).

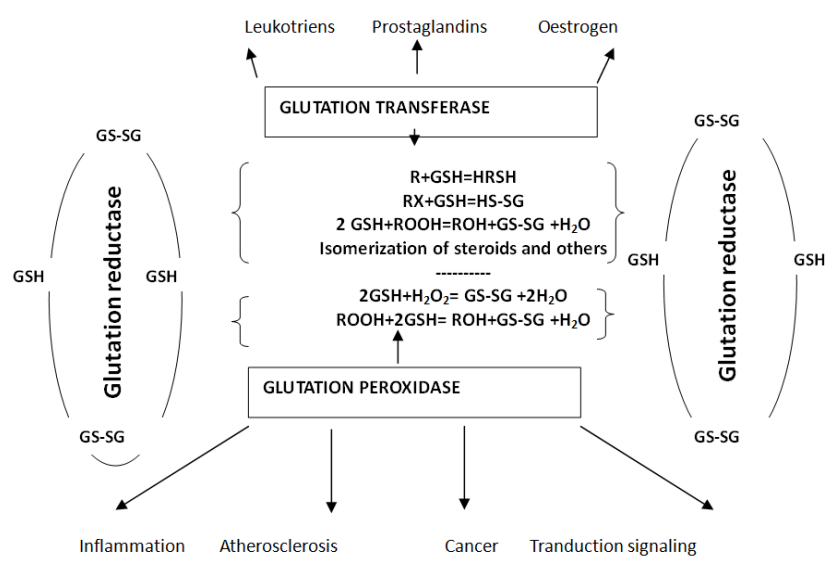

Figure 6. Diagram showing the main types of reactions of glutathione peroxidase and glutathione transferase under the fungidol influence as well as their involvement in other metabolic pathways and the role of the glutathione cycle in maintaining of redox-system in a cell.

Moreover the offset of the balance GSH $\rightarrow$ GS-SG determines the redox state of the whole cell, which can be one of the integrative systems of regulation of general metabolism. This suggests that the redox system can be considered as physical and chemical background, on which 
the metabolic pattern is implemented and this background is provided by the general reaction of cell metabolism in the rapidly changing environment (Fig. 6).

We believe that such a non-specific factor of regulation as the redox state of the cell, reflecting the peculiarities of interaction with environmental factors, is converted into a specific factor of regulation, due to such fundamental property of biological macromolecules (enzymes) as their multifunctionality. Thus, glutathione transferase, except four known enzymatic reactions is involved in the metabolism of the leukotrienes, prostaglandins, hormones, estrogen and other compounds (Fig. 6). They, in turn, are involved in a large number of other metabolic processes and form of a specific metabolic pattern.

The functions of glutathione peroxidase are not less diverse. We mention only that except the most well-known the restoration of hydroperoxides, it performs a number of specific functions. It is shown that it maintains the level of reduced glutathione, inhibits inflammation by reducing the biosynthesis of prostaglandins, inhibits the development of atherosclerosis, prevents the development of cancer [33], plays an important role in signal transduction, thereby reducing the risk of transition of physiological signal transduction to abnormal one [34], (Fig. 6).

The data available in the literature and the results of this study allow us to make a hypothesis about the mechanism of transition of nonspecific antioxidant-prooxidant system to the formation of specific adaptive metabolic patterns and manifestations of antitoxicity functions.

The essence of the hypothesis is following:

1. physical-chemical system of cells is a complex of parameters such as redox-system, osmolarity, $\mathrm{pH}$, temperature, etc., which are dynamically changed as a result of changes in exogenous and endogenous factors;

2. in spite of the endless possibilities of combination (combinatory) of physical-chemical parameters of the cellular environment, they can be grouped into a discrete set of options;

3. a possible physical-chemical embodiment will provide (inducing) expression of one or more metabolic reactions of polyfunctional enzyme systems within their structural and functional capabilities;

4. activated enzymes will form specific metabolic pattern, and the specific regulatory signals that reinforce adaptive metabolic pattern "turn on";

5. probably there is a set of universal options, which can provide a relatively wide adaptability to conditions of physical and chemical environmental factors, i.e. the formation of metabolic buffering system;

6. metabolic patterns formed in specific physical-chemical conditions of cells may provide long-term stability of the system, but may be more "specialized" and lead to disease, i.e. significantly narrow the adaptive capabilities of the system in the event of new physical and chemical conditions in the cell.

Based on the hypothesis, it is believed that antioxidant and antitoxic properties of fungidol form a common adaptive metabolic pattern appearing as interrelated phenomena.

\section{References}

[1] Barja G. (2002) Endogenous oxidative stress: relationship to aging, longevity and caloric restriction. Ageing research reviews, 1, 397-411.

[2] Celec P., Hodosy J., Pálffy, R. et al. (2013) The short-term effects of soybean intake on oxidative and carbonyl stress in men and women. Molecules, 18, 5, 5190-5120.

[3] Davydov V., Bozhkov A., Kulchitskij O. (2012) Physiological and pathophysiological role of endogenous aldehydes (modern views).-Palmarium academic publishing (Germany). $-240 \mathrm{p}$. (monograph).

[4] Harman D. (1956) Aging: a theory based on free radical biology. J. Gerontol., 11, 298-300.

[5] Emanuel N.M., Lipchina L. P. (1958) Leukemia in mice and especially its development under the influence of inhibitors of chain oxidation processes. Doklady of the Academy of Sciences of the USSR, 121, 141-144 (in Russian).

[6] Papa S., Skulachev V.P. (1997) Reactive oxygen species mitochondria, apoptosis and aging. Molec. Cell Biochem., 174, 305-319.

[7] Sohol R.S. (2002) Role of oxidative stress and protein oxidation in the aging process. Free radical Biol. Med., 33, $37-44$.

[8] Barja, G. (2004) Aging in vertebrates, and the effect of caloric restriction: a mitochondrial free radical production - DNA damage mechanism? Biol. Rev., 79, 235-251.

[9] Jia Z., Zhu H., Misra H.P., Li Y. (2008) Potent induction of total cellular GSH and NQO1 as well as mitochondrial GSH by $3 \mathrm{H}-1,2$-dithiole-3-thione in SH-SY5Y neuroblastoma cells and primary human neurons: Protection against neurocytotoxicity elicited by dopamine, 6-hydroxydopamine, 4-hydroxy-2-nonenal, or hydrogen peroxide. Brain Research, $1197,159-169$.

[10] Jobgen W.S., Fried S.K., Fu W. J. et al. (2006) Regulatory role for the arginine nitric oxide pathway inmetabolism of energy substrates. J Nutr Biochem., 17, 571-588.

[11] Skulachev V.P., Longo V.D. (2005) Aging as mitochondriamediated atavistic program. Can aging be switched off? Ann. N.Y. Acad. Sci., 1054, 145-164.

[12] Goncharova N. D., Shmaliy A. V., Bogatyrenko T. N., Koltover V. K. (2006) Correlation between activity of antioxidant enzymes and circadian rhythms of corticosteroids in Macaca mulatta monkeys of different age Experimental Gerontology Volume 41, Issue 8, 778-783.

[13] Shalaby E. A. and Sanaa M. M. S. (2013) Antioxidant compounds, assays of determination and mode of action. African Journal of Pharmacy and Pharmacology, 7(10), 528539. DOI: 10.5897/AJPP2013.3474.

[14] Koltover V.K. (2009) Bioantioxidants: the systems reliability standpoint. Toxicology and Industrial Health, 25, 295-299.

[15] Cadenas E. (2004) Mitochondrial free radical production and cell signaling. Molecular Aspects of Medicine, 25, 1-2, 17-26. 
[16] Hong-Bo Shao., Li-Ye Chu, Zhao-Hua Lu and Cong-Min Kang (2008) Primary antioxidant free radical scavenging and redox signaling pathways in higher plant cells. Int J Biol Sci. $4(1), 8-14$.

[17] Zhu X., Raina A. K., Lee H.-G., Casadesus G., Smith M. A., Perry G. (2004) Oxidative stress signalling in Alzheimer's disease. Brain Research, 1000, 1-2, 12, 32-39.

[18] Bozhkov A. I., Nikitchenko Yu.V., Sheremet A. A., Bozhkov A.A. (2014) The prooxidant-antioxidant system is the primary stage of the answer of organism on unbalanced diet. Journal of Harmonized Research in Pharmacy, 3(3), 78-90.

[19] Bozhkov A.I., and Nikitchenko Yu.V. Caloric Restriction Diet Induced Specific Epigenotypes Associated with Life Span Extension Journal of Nutritional Therapeutics, 2013, 2, 30-39.

[20] Clarkson P. M. and Thompson H. S. (2000) Antioxidants: what role do they play in physical activity and health? Am J Clin Nutr, 72, 2, 637-646.

[21] Estrada-Camarena E., Fernández-Guasti A., López-Rubalcava C. (2003) Antidepressant-like effect of different estrogenic compounds in the forced swimming test. Neuropsychopharmacology, 28 (5), 830-838.

[22] Halliwell B., Gutteridge J.M.C. and Aruoma O.I. 1987.The deoxyribosemethod: a simple "test-tube" assay for determination of rate constants of hydroxyl radical. Analitical Biochemistry, 165(1), $215-219$.

[23] Klebanov. G.I., Babenkova. I.V., Teselkin.J.O. et al. 1988. Evaluation of antioxidantactivity of blood plasma using yolk. Laboratornoe delo. 5: 59-62. (in Russian).

[24] Lankin V.Z. and Gurevich S.M. (1976) Inhibition of lipid peroxidation and detoxication of lipoperoxides by protective enzyme systems (superoxide dismutase, glutathione peroxidase and glutathione reductase) in experimental malignant growth. Doklady of Academy of Sciences of USSR 226 (3): 705-708 (in Russian).

[25] Younes M., Schlichting R. and Siegers C.- P. (1980) Glutathione-S-transferase activities in rat liver: effect of some factors influencing themetabolism of xenobiotics.
Pharmacology Research. Communs., 12, 2, 115-128.

[26] Ohkawa H., Ohahi N. and Jadi K. 1979. Assay for lipid peroxides in animal tissuesby thiobarbituric acid reaction. Analitical Biochemistry. 95 (2), 351-358.

[27] Asakawa T. and Matsushita S. (1980) Coloring condition of thiobarbituric acidtest for detecting lipid hydroperoxides. Lipids, 15(3), 137-140.

[28] A. I. Bozhkov, V. I. Sidorov, N. I. Kurguzova, V. L. Dlubovskaya (2014) Metabolic memory enhances hormesis effect to the copper ions in age-depended manner. Adv. geront., 27, 1, 72-80 (in Russian).

[29] Flora S.J.S., Saxena G., Mehta A. Reversal of lead-induced Neuronal apoptosis by Chelation Treatment in Rats: Role of reactive oxygen species and intracellular $\mathrm{Ca}^{2+}$. The Journal of pharmacology and Experimental Therapeutics, 2007, 322, 1. $108-116$.

[30] Kovaleva M.K., Menzyanova N.G., Jain A., Yadav A., Flora S.J.S., Bozhkov A.I. (2012) Effect of Hormesis in Dunaliella viridis Teodor. (Chlorophyta) Under the Influence of Copper Sulfate. International Journal on Algae, 14(1), 44-61.

[31] Gaetke L. M., Chow C. K. (2003) Copper toxicity, oxidative stress, and antioxidant nutrients. Toxicology, 189, 1-2, 147163.

[32] Boonstra J., Post J. A. (2004) Molecular events associated with reactive oxygen species and cell cycle progression in mammalian cells. Gene, 337, 1-13.

[33] Hu Ya J., and Diamond A. M. (2003) Role of Glutathione Peroxidase 1 in Breast Cancer Loss of Heterozygosity and Allelic Differences in the Response to Selenium 1 Cancer Res., 63; 3347-3351.

[34] Crack P.J., Cimdins K., Ali U., Hertzog P.J., Iannello R.C. (2006) Lack of glutathione peroxidase-1 exacerbates $A \beta$ mediated neurotoxicity in cortical neurons. Journal of Neural Transmission, 113, 5, 645-657. 